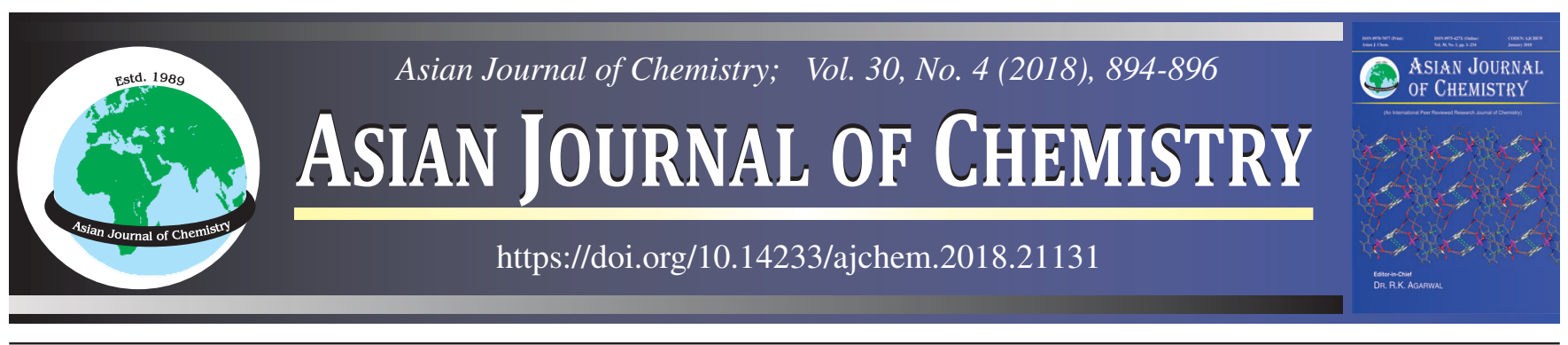

\title{
Ultrasound Assisted Synthesis of 1,5-Diaryl and 1,3,5-Triaryl-2-pyrazolines by Using KOH/EtOH System with $\mathrm{Cu}(\mathrm{I})$ Catalyst
}

Ashok S. Pise ${ }^{1}$, Sunil D. Jadhav ${ }^{2}$, Arvind S. Burungale ${ }^{3, *}$, Santosh S. Devkate ${ }^{3}$ and Ramesh B. Gawade ${ }^{3}$

${ }^{1}$ Department of Chemistry, Dada Patil Mahavidyalaya, Karjat, Ahmednagar-414 402, India

${ }^{2}$ Department of Chemistry, Mahatma Phule Arts, Science and Commerce College, Panvel-410206, India

${ }^{3}$ Department of Chemistry, S.M. Joshi College, Pune-411 028, India

*Corresponding author: E-mail: ashokpise67@gmail.com

Received: 17 November 2017; Accepted: 12 January 2018;

Published online: 28 February 2018;

AJC-18806

1,5-Diaryl-2-pyrazolines and 1,3,5-triaryl-2-pyrazolines were synthesized in alcoholic potassium hydroxide in high yields within 1-6 min
under ultrasound irradiation at room temperature.
Keywords: 1,5-Driaryl-2-pyrazolines, 1,3,5-Triaryl-2-pyrazolines, Ultrasound irradiation.

\section{INTRODUCTION}

Recently, in organic synthesis, ultrasound technique is widely used. The chemical reaction is enhanced by the ultrasonic irradiation with the help of the process of the acoustic cavitation. The reaction time is shortened by the use of ultrasonic irradiation. In the field of organic chemistry, additional convenience is offered through simple procedure of experiments, increased yields, selectivity and clean reactions of many organic transformations [1-4]. Loomis and Richards [5] first time introduced the chemical effects, which were resulted from ultrasonic irradiation in aqueous medium. There has been report of zinc$\mathrm{Cu}(\mathrm{I})$ ultrasound-mediated conjugate addition reactions developed by Luche \& co-workers which involves reactions carried out under aqueous conditions [6,7]. This methodology has been utilized in synthesis of vitamin D analogues. According to a recent trend of synthetic organic chemistry, ultrasound has been emerged as a significant tool to be used for high yield and shorter reaction time in the chemical reactions [8]. In the synthesis of natural products nitrogenous heterocycles and their derivatives are broadly applied [9]. For carbon-nitrogen bond formation, aza-Michael addition of nucleophiles to $\alpha, \beta$-unsaturated ketones, esters and nitriles is proved as an effective tool. These reactions are used in synthesis of kinase inhibitor [10], alkaloids [11], $\beta$-lactams [12], amino acids [13]. The most popular method for the preparation of 2-pyrazolines refluxing with $\alpha, \beta$-unsaturated aldehydes and ketones using phenyl hydrazine in acetic acid [14]. The substituted pyrazolines have biological activities such as antibacterial [15], analgesic
[16], anti-inflammatory [17], antiviral [18], antifungal [19], antiarthritic [20] properties. In reported method, substituted pyrazolines were synthesized using catalyst and solvent such as glacial acetic acid under heating or ultrasound irradiation [21], sodium hydroxide in ethyl alcohol under ultrasound irradiation [22], $\mathrm{K}_{2} \mathrm{CO}_{3}$-mediated microwave irradiation [23], glacial acetic acid in EtOH under microwave irradiation [24,25], EtOH [26], $\mathrm{SOCl}_{2}$ in benzene [27] under conventional heating, $\mathrm{H}_{3} \mathrm{PW}_{12} \mathrm{O}_{40}$ [28], Amberlyst 15 in refluxing toluene [29], triethanolamine [30], pyridine in refluxing ethanol [31], ethyl lactate [32] and cerium chloride heptahydrate in refluxing ethyl lactate [33]. Benzene and DMSO are the conventional solvents that have the impacts on the environment [34].

Reported methods have some limitations such as longer reaction time, poor to moderate yields, conventional method. Therefore, there is need of developing the efficient and environmental protocol for synthesis of pyrazolines derivatives. We have developed efficient, high yielding ultrasound assisted methodology for synthesis of pyrazolines derivatives using $\mathrm{KOH}$ in $\mathrm{EtOH}$ and nitratobis(triphenyl phosphine) copper(I) $\left[\mathrm{Cu}\left(\mathrm{PPh}_{3}\right)_{2} \mathrm{NO}_{3}\right]$ as a catalyst (Scheme-I). Nitratobis(triphenyl phosphine) copper(I) catalyst for the synthesis of pyrazolines is not used in the previous work.

\section{EXPERIMENTAL}

All melting points are uncorrected and were determined in a capillary tube. The IR spectra were recorded on a Shimadzu Miracle-10 ATR. The ${ }^{1} \mathrm{H}$ NMR spectra were obtained on a 


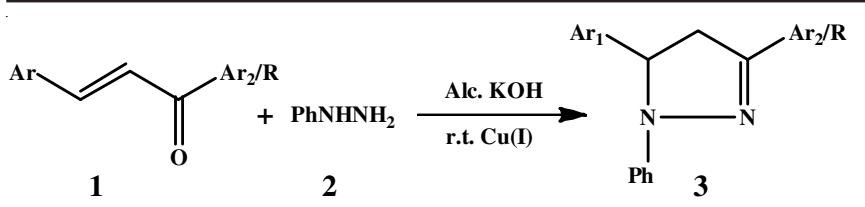

Scheme-I: General scheme of reaction

Bruker $400 \mathrm{MHz}$ spectrometer with $\mathrm{CDCl}_{3}$ as the solvent using tetramethylsilane (TMS) as the internal standard. Sonication was performed in an ultrasonic cleaner with frequency of 33 $\mathrm{KHz}$ and a normal power of $250 \mathrm{~W}$. All reactions were checked by TLC using pet, ether/ethyl acetate (8:2) as the mobile phase. The spots were visualized using UV cabinet. Crude product was purified by recrystallization using ethanol and column chromatography using pet. ether/ethyl acetate as a solvent.

Synthesis of 1,5-diaryl pyrazolines and 1,3,5-triaryl2-pyrazolines: The chalcones were prepared from acetone or acetophenone and aromatic aldehydes by reported method. Pyrazoline derivatives were prepared by dissolving chalcones $(10 \mathrm{mmol})$ and phenyl hydrazine $(10 \mathrm{mmol})$ in alcoholic $\mathrm{KOH}$ ( $1 \mathrm{~g} \mathrm{KOH}$ in $40 \mathrm{~mL}$ ethanol) in a $100 \mathrm{~mL}$ conical flask. Then $\mathrm{Cu}\left(\mathrm{PPh}_{3}\right)_{2} \mathrm{NO}_{3}$ catalyst $(10 \mathrm{~mol} \%$ i.e. $62 \mathrm{mg})$ was added in the reaction mixture. The mixture was irradiated in the water bath of an ultrasonic cleaner for the period shown in Table-2. After completion of the reaction, the reaction mixture was poured in ice water and filtered. The crude products were recrystallized in ethanol to obtained the 1,5-driaryl pyrazolines and 1,3,5triaryl-2-pyrazolines. The compounds were analyzed by FTIR and ${ }^{1} \mathrm{H}$ NMR spectroscopy.

\section{Spectral data:}

1,3,5-Triphenyl-2-pyrazoline (3a): Apricot yellow solid, m.f.: $\mathrm{C}_{21} \mathrm{H}_{18} \mathrm{~N}_{2}$, m.p.: $136^{\circ} \mathrm{C}$. IR $\left(\mathrm{KBr}, \mathrm{v}_{\max }, \mathrm{cm}^{-1}\right) 3030$ (aromatic $\mathrm{C}-\mathrm{H}), 1653(\mathrm{C}=\mathrm{N}), 1595,1489$ (aromatic C=C), ${ }^{1} \mathrm{H}$ NMR (400 $\left.\mathrm{MHz}, \mathrm{CDCl}_{3}\right) \delta 3.18(\mathrm{dd}, J=7.2,16.8 \mathrm{~Hz}, 1 \mathrm{H}), 3.88(\mathrm{dd}, J=$ $12.4,17.2 \mathrm{~Hz}, 1 \mathrm{H}), 5.32$ (dd, $J=7.2,12.4 \mathrm{~Hz}, 1 \mathrm{H}), 6.83-7.75$ $(\mathrm{m}, 15 \mathrm{H}) \mathrm{ppm}$.

1,3-Diphenyl-5-(4-nitrophenyl)-2-pyrazoline (3b): Yellow solid, m.f.: $\mathrm{C}_{21} \mathrm{H}_{17} \mathrm{~N}_{3} \mathrm{O}_{2}$, m.p.: $160^{\circ} \mathrm{C}$. IR $\left(\mathrm{KBr}, v_{\max }, \mathrm{cm}^{-1}\right) 3053$ (aromatic C-H), $1654(\mathrm{C}=\mathrm{N}), 1604,1444$ (aromatic $\mathrm{C}=\mathrm{C}), 1518$ $(\mathrm{N}-\mathrm{O}),{ }^{1} \mathrm{H}$ NMR $\left(400 \mathrm{MHz}, \mathrm{CDCl}_{3}\right) \delta 3.18(\mathrm{dd}, J=7.2,17.2$ $\mathrm{Hz}, 1 \mathrm{H}), 3.87$ (dd, $J=12.4,17.2 \mathrm{~Hz}, 1 \mathrm{H}), 5.27$ (dd, $J=7.2$, $12.8 \mathrm{~Hz}, 1 \mathrm{H}), 6.85-7.77$ (m, $14 \mathrm{H}) \mathrm{ppm}$.

1,3-Diphenyl-5-(3-chlorophenyl)-2-pyrazoline (3c): Topaz yellow solid, m.f.: $\mathrm{C}_{21} \mathrm{H}_{17} \mathrm{~N}_{2} \mathrm{Cl}$, m.p.: $133{ }^{\circ} \mathrm{C}$. IR ( $\mathrm{KBr}$, $\left.v_{\max }, \mathrm{cm}^{-1}\right) 3072$ (aromatic C-H), $1653(\mathrm{C}=\mathrm{N}), 1590,1490$ (aromatic $\mathrm{C}=\mathrm{C}),{ }^{1} \mathrm{H} \mathrm{NMR}\left(400 \mathrm{MHz}, \mathrm{CDCl}_{3}\right) \delta 3.18(\mathrm{dd}, J=$ 7.2, 17.2 Hz, 1H), 3.87 (dd, $J=12.4,17.2 \mathrm{~Hz}, 1 \mathrm{H}), 5.27$ (dd, $J=7.2,12.8 \mathrm{~Hz}, 1 \mathrm{H}), 6.85-7.77$ (m, $14 \mathrm{H}) \mathrm{ppm}$.

1,3-Diphenyl-5-(4-methoxyphenyl)-2-pyrazoline (3d): Orange solid, m.f.: $\mathrm{C}_{22} \mathrm{H}_{20} \mathrm{~N}_{2} \mathrm{O}$, m.p.: $110{ }^{\circ} \mathrm{C}$. IR (KBr, $v_{\max }$, $\left.\mathrm{cm}^{-1}\right) 3059$ (aromatic C-H), $1656(\mathrm{C}=\mathrm{N}), 1595,1492$ (aromatic $\mathrm{C}=\mathrm{C}),{ }^{1} \mathrm{HNMR}\left(400 \mathrm{MHz}, \mathrm{CDCl}_{3}\right) \delta 3.18(\mathrm{dd}, J=7.2,17.2 \mathrm{~Hz}$, $1 \mathrm{H}), 3.87$ (dd, $J=12.0,16.8 \mathrm{~Hz}, 1 \mathrm{H}), 3.87$ (s, 3H) 5.28 (dd, $J$ $=7.2,12.4 \mathrm{~Hz}, 1 \mathrm{H}), 6.80-7.77(\mathrm{~m}, 14 \mathrm{H}) \mathrm{ppm}$.

1,3-Diphenyl-5-(3-nitrophenyl)-2-pyrazoline (3e): Peach yellow solid, m.f.: $\mathrm{C}_{21} \mathrm{H}_{17} \mathrm{~N}_{3} \mathrm{O}_{2}$, m.p.: $134^{\circ} \mathrm{C}$. IR (KBr, $v_{\max }$, $\mathrm{cm}^{-1}$ ) 3061 (aromatic C-H), $1656(\mathrm{C}=\mathrm{N}), 1593,1489$ (aromatic $\mathrm{C}=\mathrm{C}), 1480(\mathrm{~N}-\mathrm{O}),{ }^{1} \mathrm{H}$ NMR $\left(400 \mathrm{MHz}, \mathrm{CDCl}_{3}\right) \delta 3.16(\mathrm{dd}$,
$J=7.2,17.2 \mathrm{~Hz}, 1 \mathrm{H}), 3.90(\mathrm{dd}, J=12.4,17.2 \mathrm{~Hz}, 1 \mathrm{H}), 5.30$ (dd, $J=7.2,12.0 \mathrm{~Hz}, 1 \mathrm{H}), 6.82-7.76(\mathrm{~m}, 14 \mathrm{H}) \mathrm{ppm}$.

1,5-Diphenyl-3-methyl-2-pyrazoline (3f): Lemon yellow solid, m.f.: $\mathrm{C}_{16} \mathrm{H}_{16} \mathrm{~N}_{2}$, m.p.: $150{ }^{\circ} \mathrm{C}$. IR ( $\left.\mathrm{KBr}, v_{\max }, \mathrm{cm}^{-1}\right) 3026$ (aromatic C-H), $1660(\mathrm{C}=\mathrm{N}), 1593,1496$ (aromatic $\mathrm{C}=\mathrm{C}),{ }^{1} \mathrm{H}$ NMR $\left(400 \mathrm{MHz}, \mathrm{CDCl}_{3}\right) \delta 1.59(\mathrm{~s}, 3 \mathrm{H}) 3.08(\mathrm{dd}, J=6.8,16.8$ $\mathrm{Hz}, 1 \mathrm{H}), 3.78$ (dd, $J=12.4,16.8 \mathrm{~Hz}, 1 \mathrm{H}), 5.31$ (dd, $J=6.8,12.4$ $\mathrm{Hz}, 1 \mathrm{H}), 6.54-7.35(\mathrm{~m}, 10 \mathrm{H}) \mathrm{ppm}$.

1-Phenyl-5-(4-chlorophenyl)3-methyl-2-pyrazoline (3g): Lemon yellow solid, m.f.: $\mathrm{C}_{16} \mathrm{H}_{15} \mathrm{~N}_{2} \mathrm{Cl}$, m.p.: $160{ }^{\circ} \mathrm{C}$. IR $\left(\mathrm{KBr}, v_{\max }, \mathrm{cm}^{-1}\right) 3026$ (aromatic C-H), $1651(\mathrm{C}=\mathrm{N}), 1597$, 1496 (aromatic C=C), ${ }^{1} \mathrm{H}$ NMR $\left(400 \mathrm{MHz}, \mathrm{CDCl}_{3}\right) \delta 1.63$ (s, $3 \mathrm{H}), 3.01(\mathrm{dd}, J=6.8,16.8 \mathrm{~Hz}, 1 \mathrm{H}), 3.75(\mathrm{dd}, J=12.4,16.8 \mathrm{~Hz}$, 1H), 5.29 (dd, $J=6.4,12.0 \mathrm{~Hz}, 1 \mathrm{H}), 6.48-7.39$ (m, $9 \mathrm{H}) \mathrm{ppm}$.

1,3-Diphenyl-5-(3-bromophenyl)-2-pyrazoline $(3 \mathrm{~h})$ : Pale yellow solid, m.f.: $\mathrm{C}_{21} \mathrm{H}_{17} \mathrm{~N}_{2} \mathrm{Br}$, m.p.: $140{ }^{\circ} \mathrm{C}$. IR $(\mathrm{KBr}$, $\left.v_{\max }, \mathrm{cm}^{-1}\right) 3030$ (aromatic C-H), $1654(\mathrm{C}=\mathrm{N}), 1602,1498$ (aromatic $\mathrm{C}=\mathrm{C}),{ }^{1} \mathrm{H}$ NMR $\left(400 \mathrm{MHz}, \mathrm{CDCl}_{3}\right) \delta 3.05(\mathrm{dd}, J=$ 6.8, 17.2 Hz, 1H), 3.35 (dd, $J=12.4,17.2 \mathrm{~Hz}, 1 \mathrm{H}), 5.63$ (dd, $J=6.8,12.4 \mathrm{~Hz}, 1 \mathrm{H}), 6.79-7.70(\mathrm{~m}, 14 \mathrm{H}) \mathrm{ppm}$.

\section{RESULTS AND DISCUSSION}

The effect of reaction conditions on the formation of substituted pyrazolines under ultrasound irradiation was summarized in Table-1.

TABLE-1

EFFECT OF REACTION CONDITION ON SYNTHESIS OF PYRAZOLINES UNDER ULTRASOUND IRRADIATION

\begin{tabular}{cccc}
\hline Entry & Base & $\begin{array}{c}\text { Molar ratio of } \\
\text { EtOH: } \mathrm{H}_{2} \mathrm{O}\end{array}$ & $\begin{array}{c}\text { Isolated yield } \\
(\%)\end{array}$ \\
\hline 3a & $\mathrm{LiOH}$ & $\mathrm{EtOH}$ & 0 \\
3a & $\mathrm{K}_{2} \mathrm{CO}_{3}$ & $\mathrm{EtOH}$ & 0 \\
3b & $\mathrm{KOH}$ & $\mathrm{H}_{2} \mathrm{O}$ & 0 \\
3c & $\mathrm{KOH}$ & $\mathrm{EtOH}: \mathrm{H}_{2} \mathrm{O} 1: 3$ & Trace \\
3d & $\mathrm{KOH}$ & $\mathrm{EtOH}: \mathrm{H}_{2} \mathrm{O} 1: 1$ & 35 \\
3e & $\mathrm{KOH}$ & $\mathrm{EtOH}: \mathrm{H}_{2} \mathrm{O} 3: 1$ & 65 \\
3f & $\mathrm{KOH}$ & $\mathrm{EtOH}$ & 91 \\
\hline
\end{tabular}

When we performed the reaction using other bases such as $\mathrm{LiOH}, \mathrm{K}_{2} \mathrm{CO}_{3}$, no product (Table-1, 3a) was obtained. It is also important to mention that when reaction was performed in water (Table-1, 3b) no product was obtained. The substrates chalcones and phenyl hydrazine are insoluble in water at room temperature. Therefore reaction does not proceeded in water. When the molar ratio of chalcones and phenyl hydrazine was 1:1 the yield of 2-pyrazoline (Table-2 entry, 3a-3h) was obtained in $97 \%$ yield. The increasing molar ratio of $\mathrm{EtOH}: \mathrm{H}_{2} \mathrm{O}$ the yield of 2-pyrazoline (Table-1, 3e) was increased. It is apparent that the reaction can be finished in shorter time to give excellent yield under ultrasound. However, for the substrate possessing electron withdrawing groups (Table-2, 3b, 3e) excellent yields were obtained. The electron withdrawing and donating substituted aldehydes are used in synthesis of substituted pyrazolines and there is no effect on yield of the product. The $\mathrm{KOH}$ is the base, ethanol as a reaction solvent and $\mathrm{Cu}\left(\mathrm{PPh}_{3}\right)_{2} \mathrm{NO}_{3}$ is the catalyst.

In nitratobis(triphenyl phosphine) copper(I) $\left(\mathrm{Cu}\left(\mathrm{PPh}_{3}\right)_{2} \mathrm{NO}_{3}\right)$ reaction proceeded smoothly under ultrasound irradiation and water is not efficient solvent for the synthesis of 2-pyrazolines. 


\begin{tabular}{|c|c|c|c|c|c|c|}
\hline \multicolumn{7}{|c|}{$\begin{array}{l}\text { TABLE-2 } \\
\text { SYNTHESIS OF 1,5-DRIARYL-2-PYRAZOLINES AND 1,3,5-TRIARYL-2-PYRAZOLINES IN } \\
\text { ALCOHOLIC KOH UNDER ULTRASOUND IRRADIATION AT ROOM TEMPERATURE }\end{array}$} \\
\hline Entry & $\mathrm{Ar}_{1}$ & $\mathrm{Ar}_{2} / \mathrm{R}$ & Time (min) & Yield (\%) & m.p. (found) $\left({ }^{\circ} \mathrm{C}\right)$ & Ref. \\
\hline $\mathbf{3 a}$ & $\mathrm{C}_{6} \mathrm{H}_{5}$ & $\mathrm{C}_{6} \mathrm{H}_{5}$ & 1 & 97 & $136(134-135)$ & {$[21]$} \\
\hline $3 \mathbf{b}$ & $4-\mathrm{NO}_{2} \mathrm{C}_{6} \mathrm{H}_{4}$ & $\mathrm{C}_{6} \mathrm{H}_{5}$ & 6 & 97 & $160(156-158)$ & [33] \\
\hline $3 c$ & $3-\mathrm{ClC}_{6} \mathrm{H}_{4}$ & $\mathrm{C}_{6} \mathrm{H}_{5}$ & 5 & 95 & $134(134-135)$ & [21] \\
\hline 3d & $4-\mathrm{CH}_{3} \mathrm{OC}_{6} \mathrm{H}_{4}$ & $\mathrm{C}_{6} \mathrm{H}_{5}$ & 6 & 92 & $110(110-111)$ & [21] \\
\hline $3 e$ & $3-\mathrm{NO}_{2} \mathrm{C}_{6} \mathrm{H}_{4}$ & $\mathrm{C}_{6} \mathrm{H}_{5}$ & 2 & 95 & $134(133-135)$ & [21] \\
\hline 3f & $\mathrm{C}_{6} \mathrm{H}_{5}$ & $\mathrm{CH}_{3}$ & 2 & 91 & 150 & \\
\hline $3 g$ & 4- $\mathrm{ClC}_{6} \mathrm{H}_{4}$ & $\mathrm{CH}_{3}$ & 1 & 90 & 161 & \\
\hline $3 \mathbf{h}$ & $3-\mathrm{BrC}_{6} \mathrm{H}_{4}$ & $\mathrm{C}_{6} \mathrm{H}_{5}$ & 5 & 88 & $140(141-142)$ & [21] \\
\hline
\end{tabular}

From Table-2, it is suggested that using catalyst such as $\mathrm{Cu}\left(\mathrm{PPh}_{3}\right)_{2} \mathrm{NO}_{3}$ shortened the reaction time of the preparation of pyrazolines derivatives from $2 \mathrm{~h}$ under classical conditions to $6 \mathrm{~min}$. In addition, the yields of the products were improved by $10-15 \%$ in comparison with those obtained by the previous method.

\section{Conclusion}

In conclusion, we found that 1,5-driaryl-2-pyrazolines and 1,3,5-triaryl-2-pyrazolines were synthesized from acetone or acetophenone and substituted aldehydes with phenyl hydrazine in alcoholic $\mathrm{KOH}$ using $\mathrm{Cu}\left(\mathrm{PPh}_{3}\right)_{2} \mathrm{NO}_{3}$ as a catalyst under ultrasound irradiation to obtained excellent yields. The short reaction time, easy work up and high yields makes this catalyst a more convenient alternative to the reported catalysts.

\section{ACKNOWLEDGEMENTS}

The author thanks the Central Instrumentation Facility, Savitribai Phule Pune University, Pune, India for the spectral analysis.

\section{REFERENCES}

1. T.J. Mason and J.P. Lorimer, Sonochemistry: Theory, Application and Uses of Ultrasound in Chemistry. Wiley, New York (1988).

2. A. Gaplovsky, M. Gaplovsky, S. Toma and J.L. Luche, J. Org. Chem., 65, 8444 (2000);

https://doi.org/10.1021/jo000611+.

3. R.R. Deshmukh, R. Rajagopal and K.V. Srinivasan, Chem. Commun., 17, 1544 (2001); https://doi.org/10.1039/b104532f.

4. G. Cravotto and P. Cintas, Chem. Soc. Rev., 35, 180 (2006); https://doi.org/10.1039/B503848K.

5. W. Richards and A. Loomis, J. Am. Chem. Soc., 49, 3086 (1927); https://doi.org/10.1021/ja01411a015.

6. L.A. Sarandeses, A. Mouriño andJ.-L. Luche, J. Chem. Soc. Chem. Commun., 798 (1992);

https://doi.org/10.1039/C39920000798.

7. C. Dupuy, C. Petrier, L.A. Sarandeses and J.L. Luche, Synth. Commun., 21, 643 (1991);

https://doi.org/10.1080/00397919108020831.

8. V. Bejan, C. Moldoveanu and I.I. Mangalagiu, Ultrason. Sonochem., 16, 312 (2009); https://doi.org/10.1016/j.ultsonch.2008.10.012.

9. A.R. Katritzky and A.F. Pozharskii, Handbook of Heterocyclic Chemistry, Pergamon, Oxford, edn 2 (2002).

10. Q. Lin, D. Meloni, Y. Pan, M. Xia, J. Rodgers, S. Shepard, M. Li, L. Galya, B. Metcalf, T.-Y. Yue, P. Liu and J. Zhou, Org. Lett., 11, 1999 (2009);

https://doi.org/10.1021/o1900350k.
11. S. Fustero, S. Monteagudo, M. Sánchez-Roselló, S. Flores, P. Barrio and C. del Pozo, Chem. Eur. J., 16, 9835 (2010); https://doi.org/10.1002/chem.201000615.

12. S. Comesse, M. Sanselme and A. Daïch, J. Org. Chem., 73, 5566 (2008); https://doi.org/10.1021/jo702752w.

13. K. Damera, K.L. Reddy and G.V.M. Sharma, Lett. Org. Chem., 6, 151 (2009); https://doi.org/10.2174/157017809787582834

14. A. Levai, ARKIVOC, 344 (2005); https://doi.org/10.3998/ark.5550190.0006.929.

15. A.M. Fahmy, A.A. Hassa and R.A. Ahmed, Indian J. Chem., 26B, 884 (1987).

16. R.A. Nugent, M. Murphy, S.T. Schlachter, C.J. Dunn, R.J. Smith, L.A. Staite, L.A. Galinet, S.K. Shields, D.G. Aspar, K.A. Richard and N.A. Rohloff, J. Med. Chem., 36, 134 (1993); https://doi.org/10.1021/jm00053a017.

17. V. Rangari, V.N. Gupta and C.K. Atal, Indian J. Pharm. Sci., 52, 158 (1990).

18. M.I. Husain and S. Shukla, Indian J. Chem., 25B, 983 (1986).

19. S. Rich and J.G. Horsfall, Chem. Abstr., 46, 11543 (1952).

20. H. Kawazura, Y. Takahashi, Y. Shiga, F. Shimada, N. Ohto and A. Tamura, Jpn. J. Pharmacol., 73, 317 (1997); https://doi.org/10.1254/jip.73.317.

21. Z.-P. Lin and J.-T. Li, E-J. Chem., 9, 267 (2012); https://doi.org/10.1155/2012/364798.

22. R. Gupta, N. Gupta and A. Jain, Indian J. Chem., 49B, 351 (2010).

23. M. Kidwai, S. Kukreja and R. Thakur, Lett. Org. Chem., 3, 135 (2006); https://doi.org/10.2174/157017806775224170.

24. M.A.H. Zahran, H.F. Salama, Y.G. Abdin and A.M. Gamal-Eldeen, J. Chem. Sci., 122, 587 (2010); https://doi.org/10.1007/s12039-010-0093-9.

25. A. Goyal, S. Sharma and J. Gaba, Indian J. Chem., 56B, 334 (2017).

26. V. Malhotra, S. Pathak, R. Nath, D. Mukerjee and K. Shankar, Indian J. Chem., 41B, 1310 (2002).

27. A. Tiwari, S. Fatma A. Bishnoi, A. Srivastava and B. Banerjee, Indian J. Chem., 56B, 317 (2017).

28. R. Fazaeli, Open Catalysis J., 3, 79 (2010); https://doi.org/10.2174/1876214X01003010079.

29. B. Holla and M. Mahalinga, Indian J. Chem., 45B, 568 (2002).

30. S. Mokle, A. Vibhute S. Khansole, S. Zangade and Y. Vibhute, Res. J. Pharm. Biol. Chem. Sci., 1, 631 (2010).

31. S. Sridhar and Y. Rajendraprasad, E-J. Chem., 9, 1810 (2012); https://doi.org/10.1155/2012/476989.

32. S. Paul, K. Pradhan and A.R. Das, Green Chem., 3, 111 (2016); https://doi.org/10.2174/2213346103666151203203139.

33. P. Bhat, G. Shridhar, S. Ladage and L. Ravishankar, J. Chem. Sci., 129, 1441 (2017); https://doi.org/10.1007/s12039-017-1327-x.

34. N. Uzma, B. Khaja Mohinuddin Salar, B. Kumar, N. Aziz, M. David and V. Reddy, Int. J. Environ. Res. Public Health, 5, 139 (2008); https://doi.org/10.3390/ijerph5030139. 